\title{
Optimal Arrangement of Wearable Devices Based on Lifespan of Animals as Device Transporter Materials for Long-term Monitoring of Wildlife Animal Sensor Network
}

\author{
Keijiro Nakagawa ${ }^{*}$ and Hill Hiroki Kobayashi \\ Center for Spatial Information Science, The University of Tokyo, \\ 5-1-5 Kashiwanoha, Kashiwa-shi, Chiba 277-8568, Japan
}

(Received August 23, 2019; accepted November 18, 2019)

Keywords: wearable device, wireless sensor, long-term monitoring, wild animal, lifespan

In this paper, we discuss the optimal arrangement of wearable devices based on the lifespan of animals, which are utilized as device transporter materials, for the long-term monitoring of the wildlife animal sensor network using multi-agent simulation to reproduce the wild environment. Related studies have proposed animal-to-animal data sharing to realize longterm monitoring in the wild environment. The research on the power saving of wireless communication in a group of animals has been conducted using animal behavior. However, a system that considers the lifespan of transported wildlife, in addition to power saving, is required for long-term monitoring in the wild environment. Herein, we used multi-agent simulation to reproduce the wild environment considering the lifespan of animals, and examined when and how many individuals should be introduced to the wild environment to realize data sharing in the group of animals over several years. The proposed method is suggested to enable the long-term monitoring of remote areas where people cannot enter without base stations and power infrastructure using wild animals for several years.

\section{Introduction}

Several methods have been used to monitor the wild environment. For example, in addition to the method where a surveyor periodically visits the wild environment, environmental monitoring in remote areas has been conducted with the evolution of technology by installing fixed sensors, including voice microphones, to acquire data, such as temperature and humidity, in the wild environment. However, the workload for surveyors in the field is extremely high, particularly in large wild environments that are difficult for people to enter. Moreover, the installation of fixed sensors mentioned earlier is also challenging because of power and communication infrastructure limitations. In particular, the area around a nuclear power plant is still designated as an evacuation area where people cannot enter given the risk of radiation leak, such as the Fukushima Daiichi Nuclear Power Plant accident, which was attributed to the Great East Japan Earthquake of March 2011. On the other hand, the effects of radioactive contamination in the ecosystem, which require long-term surveys, are of significant concern. ${ }^{(1)}$ 
Therefore, a low-workload method is required to obtain environmental information in areas without an electricity or communication infrastructure.

\section{Related Study}

\subsection{Wild animals as transporter materials}

To obtain environmental information in areas where power and communication infrastructures are limited, the use of sensors attached to various materials is being investigated. For example, in the survey of a bridge girder in an urban environment, a fixed acceleration sensor was installed on the bridge girder, and the real-time monitoring of the characteristics was conducted using the collected vibration data. ${ }^{(2)}$ In addition, a method using several vehicles equipped with sensors has been investigated as an indirect method of collecting information in an urban environment given that vehicles are readily available and can be moved into the urban environment as mobile materials. ${ }^{(3)}$

On the other hand, collecting environmental information in the natural environment, such as temperature and humidity, is generally conducted using fixed sensors, including voice microphones and cameras, as part of wildlife environmental assessment. For instance, the effects of artificial structures, such as dams, on wildlife have been investigated. However, it is challenging to sufficiently increase the number of fixed sensors when monitoring the natural environment owing to power and communication infrastructure limitations. This increases the difficulty in collecting environmental information given the large area to be surveyed. Therefore, to adequately monitor the natural environment, we will focus on attaching wearable devices to wildlife as mobile materials that can be moved in the natural environment to collect environmental information.

\subsection{Wild animals and wearable devices}

To acquire environmental information in areas without power and communication infrastructures, the use of a wearable device attached to a wild animal has been investigated as a method of acquiring environmental information. ${ }^{(4)}$ The macro-perspective investigation of the wild environment is possible by analyzing satellite image data, which is one of the remote sensing technologies. However, micro-perspective investigation, as a survey method of the area where people cannot rapidly enter, is challenging given that detailed data, such as radiation for each location, are required to conduct a sufficient survey. On the other hand, attaching a wearable device to a wild animal is one of the methods of collecting environmental information, such as temperature and radiation. Eventually, the wild animals wearing a device are captured in the area where people can enter, and the data are collected and stored. A real-time clock and GPS allow the timestamp and location of the sensor data to be obtained; therefore, researchers can analyze detailed data for each location. However, given that the capture of each wild animal is a prerequisite for data collection in the wild environment, the surveyor faces a high load of capturing the wild animals wearing a device in areas where people cannot access, which is the subject of this paper (see Fig. 1). 


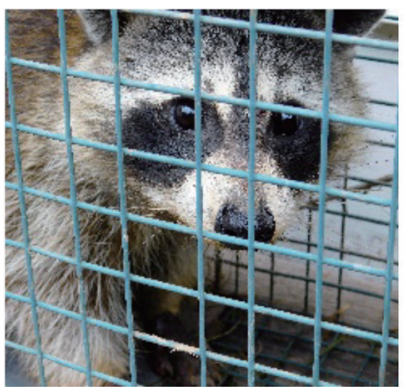

(a)

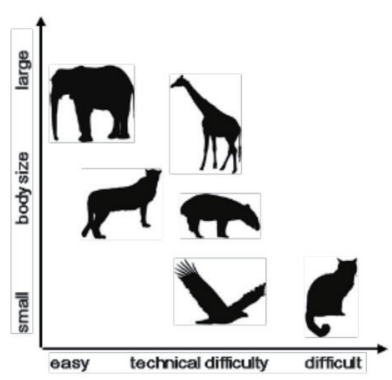

(b)

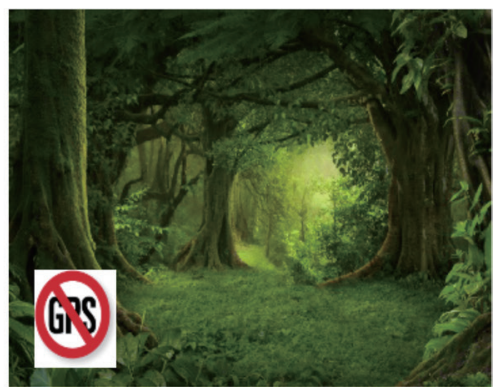

(c)

Fig. 1. (Color online) (a) Wild animal captured in a trap, (b) diagram showing the relationship between body size and technical difficulty, and (c) illustration showing that GPS and mobile signals typically do not work within a forest. ${ }^{\left({ }^{(8)}\right.}$

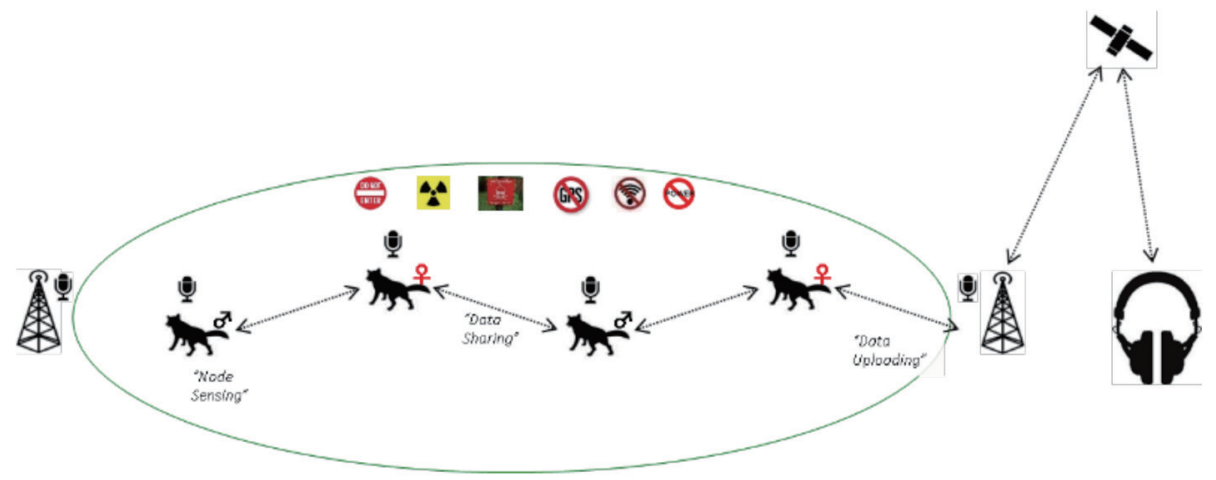

Fig. 2. (Color online) Proposed sensing system/concept. ${ }^{(8)}$

\subsection{Animal-to-animal data sharing mechanism}

To solve these problems, an animal-to-animal data sharing mechanism has been proposed in Fig. 2..$^{(5,6)}$ Using a wearable device equipped with wireless communication, Geiger counter, real-time clock, and GPS function systems, each wild animal senses and acquires environmental information from each sensor. When the wild animals gather in a group, wireless communication is used to transfer data between each individual using a multi-hop communication or store-and-forward mechanism. To collect data from wild animals wearing a device, we proposed a mechanism that sets up traps for wildlife and uploads data to a server using wireless communication from an individual who triggered the trap. Using this approach, the surveyor does not need to capture the wild animals to collect the data acquired in the wild environment.

\subsection{Research for power saving}

However, given that this method uses wireless communication to transfer data, power consumption is high, while the mountable battery capacity is limited. Therefore, battery 
replacement or battery power saving has to be considered. Battery replacement is not an effective method because it requires the capture of an individual with a device. Therefore, in this study, multi-hop communication has been proposed, which uses wild animal behavior for power saving as shown in Fig. 3. By this method, field investigation is made possible by performing data propagation while suppressing power consumption. In previous research, field experiments using four dogs bred at Azabu University and some other field experiments using livestock cattle around the Fukushima Daiichi nuclear accident were conducted as shown in Fig. $4 .^{(7,8)}$ Therefore, the effectiveness of the power saving method in animal-to-animal data sharing has been partially verified and its research for power saving is in progress.

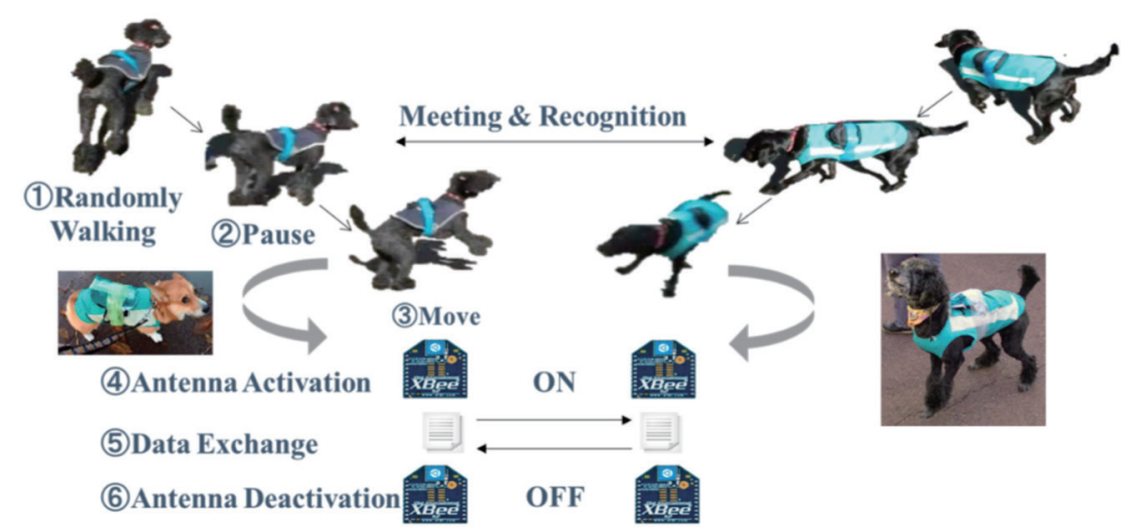

(a)

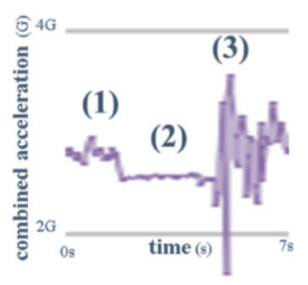

Concerned Behavior

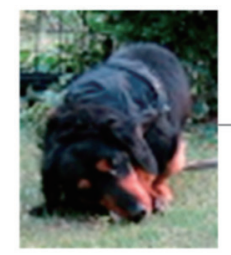

(1) Move

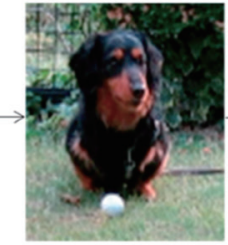

(2) Pause

(b)

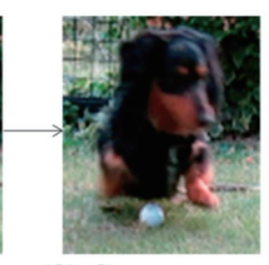

(3) Strenuous

Movement

Fig. 3. (Color online) (a) Animal-to-animal data sharing process and (b) reaction state of dogs to the peripheral. ${ }^{(7)}$

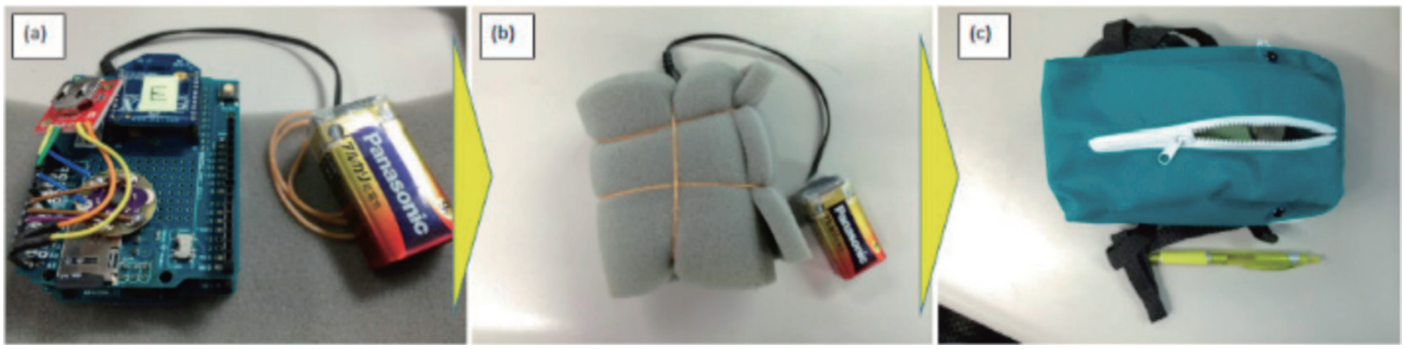

Fig. 4. (Color online) (a) Animal-to-animal data sharing prototype, (b) covered by sponge for protection from shocks, and (c) placed in the bag. ${ }^{(7)}$ 


\subsection{Problem statement: lifespan of individuals under wild environment}

Nevertheless, the system development considering power saving as shown in previous research is not sufficient in terms of long-term monitoring in the wild environment. The operating period of the wild animals as transporter materials needs to consider the power capacity of the battery of the wearable device and the lifespan of the wild animals. Given that the technology of the battery capacity has improved significantly, the lifespan of the individual is assumed to be shorter than the battery life. This is attributed to the early death of the individual given its exposure to various threats, such as traffic accidents, capture, predators, infectious diseases, and deep winter, as characteristics of the wild environment. Therefore, long-term monitoring requires the consideration of the battery life and individual lifespan.

There are two main approaches to long-term monitoring that takes lifespan into account. The first approach is a method of selecting a young individual, such as a juvenile, as the mounting target of a wearable device. When the target individual is a juvenile, there is an advantage in longevity compared with adult animals. However, the battery mounting size is limited given that the size of the individual's body is small until it becomes an adult, which does not make it an effective method. In addition, owing to the significant changes in the body size of a juvenile to become an adult with aging, the removal of the attached wearable device may occur.

The second approach is to introduce regularly several individuals to the wild environment. Given that wild animals have a lifespan, it is necessary to continuously introduce additional animals in the wild environment to maintain long-term animal-to-animal data sharing. However, increasing the population infinitely is challenging because it creates a high workload for the surveyors. Therefore, it is essential to consider when and how many individuals should be introduced to the wild environment considering the time of death of each individual.

\section{Proposed Method}

In this paper, we used multi-agent simulation (artisoc) ${ }^{(9,10)}$ to reproduce the wild environment and examine when and how many individuals should be introduced to the wild environment to realize data sharing based on the group behavior over several years. As a result, we have identified the most efficient method with the largest number of encounters exhibiting group behavior, although the workload to introduce individuals is similar to other methods. Figure 5 is an example showing a wild environment and represents a plurality of raccoons wearing wearable devices. In Fig. 5(a), the number of individuals wearing wearable devices decreases with the lapse of time, and all individuals die after 1.5 years given their lifespan. In this case, the acquired data of each individual is not transmitted to other individuals and will be lost without transferring it to the surveyors. On the other hand, in Fig. 5(b), although the number of individuals temporarily decreases, we found that the number of individuals wearing wearable devices was maintained given that individuals were periodically introduced. Therefore, the data acquired by each individual is propagated to the newly introduced individuals in the wild environment. Therefore, the proposed method enables the long-term monitoring of the wild environment without being affected by the lifespan of individuals. 


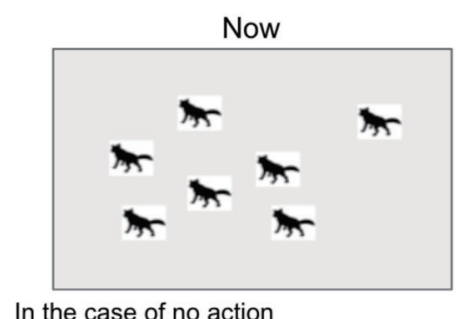

In the case of no action

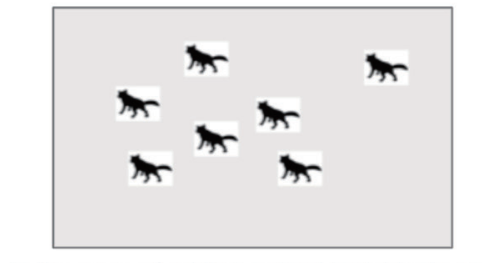

In the case of adding animal individuals periodically

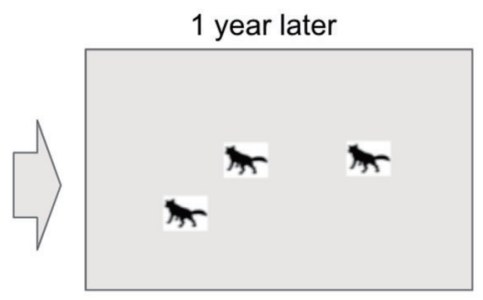

(a)

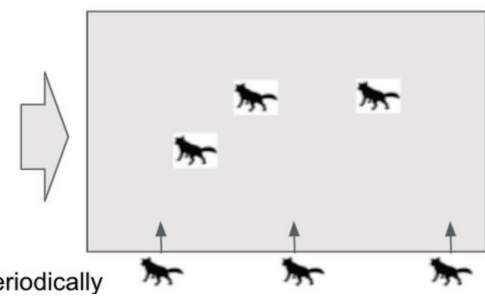

(b)
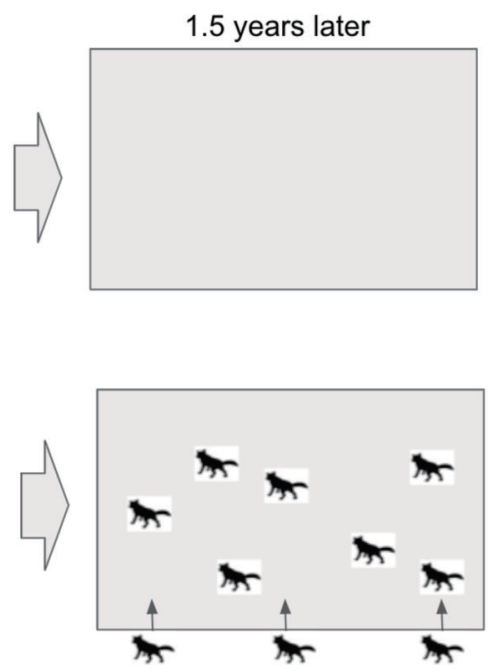

Fig. 5. State transition of density of wild animals in the cases of (a) no action and (b) adding individuals periodically.

In this paper, we examined four methods with different conditions in terms of the "number of individuals introduced at one time" and the "number of injections." Animal-to-animal data sharing can be realized by the large number of individuals and the number of frequent inputs as much as possible. However, it is impossible to increase the number of individuals without limit because of the corresponding increase in the workload of the investigators. In this paper, we assumed that a total of 350 animals were introduced to the wild environment in approximately three years following the result of the field survey. ${ }^{(11)}$ Simulations considering the number of individuals introduced at one time and the number of injections are performed under the following four conditions in Table 1 to clarify the efficient method with a high number of encounters in the long-term monitoring beyond the lifespan of each individual.

\section{Evaluation and Results}

\subsection{Evaluation on multi-agent simulation}

Each method discussed in the previous section was verified using "artisoc," which is a multi-agent simulation platform. ${ }^{(10)}$ A multi-agent system is a mechanism that can analyze a dynamically changing society by defining action rules and the interactions of agents acting autonomously and constructing an artificial society in a computer. ${ }^{(9,12,13)}$ Furthermore, artisoc is a platform for multi-agent simulation with an intuitive operation that can rapidly reflect ideas into a model (Fig. 6).

Given that the related research was targeting raccoons supposed to be wearing wearable devices, in this paper, we also targeted raccoons using biological information obtained from long-time raccoon surveys in the wild environment by Rosatte et al. ${ }^{(11)}$ We set up the parameters of raccoons and the environment in Table 2 following the results of Rosatte et al. ${ }^{(11)}$ 
Table 1

Parameters of each method.

\begin{tabular}{lcc}
\hline & Number of individuals & Number of times \\
\hline Method 1 & 0 & 0 \\
Method 2 & 175 & 2 (per twelve months) \\
Method 3 & 70 & 5 (per six months) \\
Method 4 & 10 & 35 (per one months) \\
\hline
\end{tabular}

Table 2

(a) Agent parameters based on biological research for raccoon.

\begin{tabular}{lc}
\hline \multicolumn{2}{c}{ Agent parameters } \\
\hline Age & 18 months \\
Maximum life & 18 months \\
Speed & $55.9 \mathrm{~m} / \mathrm{h}$ \\
Direct distance & $1.54 \mathrm{~km}$ \\
Distance of encounter & Less than $50 \mathrm{~m}$ \\
Direction & Randomly changing \\
& (per 1.54 km they move) \\
\hline
\end{tabular}

(b) General parameters in artisoc.

\begin{tabular}{lc}
\hline \multicolumn{2}{c}{ General parameters } \\
\hline Area & $10 \times 10 \mathrm{~km}^{2}$ \\
Density of individuals & 350 (initial status) \\
1 Step (artisoc) & $10 \mathrm{~min}$ \\
\hline
\end{tabular}

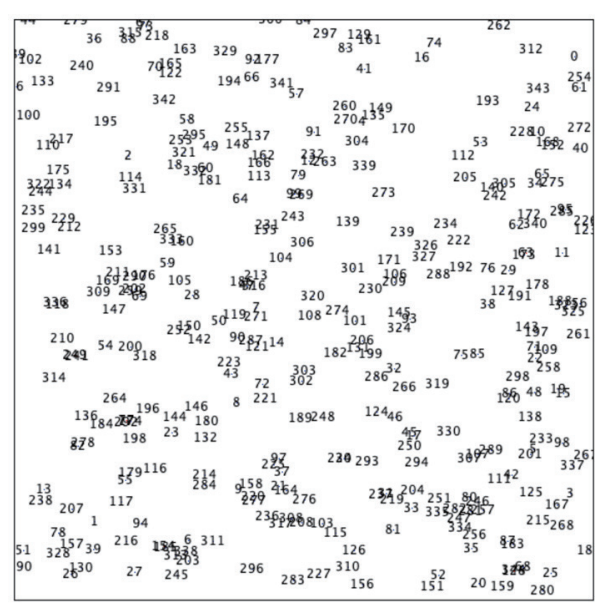

Fig. 6. Multi-agent simulation system by artisoc (10 $\left.\times 10 \mathrm{~km}^{2}\right)$.

The area of the wild environment in the simulation is $100 \mathrm{~km}^{2}\left(10 \times 10 \mathrm{~km}^{2}\right)$, and the initial population of raccoons is 350 individuals, which conform to the density of the survey results. In the wild environment on simulation, when an individual encounters another individual, communication between animals is considered to be performed if their radius is within $50 \mathrm{~m}$. The simulation period is 3 years, which is the lifespan of the raccoon in the wild environment. The individual wearing the device is assumed to be an adult animal of approximately 1.5 years or older; therefore, the initial wearing individual will end its life in 1.5 years. If the simulation period is three years, sufficient knowledge can be obtained by comparing and examining the number of individuals introduced at one time and the number of injections, which we want to clarify in this paper.

On the other hand, in this simulation, no consideration is given to the individual territory, age, and gender of each condition that may affect the results of the simulation. Although the individual territory is essential in considering the range of behavior that may directly affect the number of encounters, it is excluded from the experimental conditions because it cannot be assumed as an experimental condition owing to insufficient findings of the individual territory. It is also known to change depending on the size of the habitat. ${ }^{(1)}$ In addition, although it is known that the distance and speed an individual moves daily differ depending on age and sex, this condition is also excluded from the experimental conditions in this study because of insufficient knowledge regarding the distributions of age and sex in the habitat. 


\subsection{Results}

The results for the number of encounters differed depending on the conditions following the four methods when the total number of 350 animals in three years was divided into the conditions in terms of the number of individuals introduced at one time and the number of injections in Figs. 7 and 8. The results for the number of encounters for each method show that "Method 4" has the lowest number of individuals introduced at one time and the highest number of injections at approximately 1.69 times compared with "Method 1" without the addition of individual and injection. Moreover, "Method 4" was approximately 1.11 times more than "Method 2" with the highest number of individuals introduced at one time and the smallest number of injections in Fig. 9. Therefore, the method of increasing the number of injections rather than that of increasing the number of individuals introduced at one time is efficient for increasing the number of encounters, which is the number of wireless communications between animals, when introducing the same number of individuals in three years.

\section{Discussion}

\subsection{Forecast of the system considering the lifespan of individuals in the wild environment}

In this paper, we have shown the most efficient method of periodically introducing individuals to the wild environment considering the lifespan of the individual. As discussed

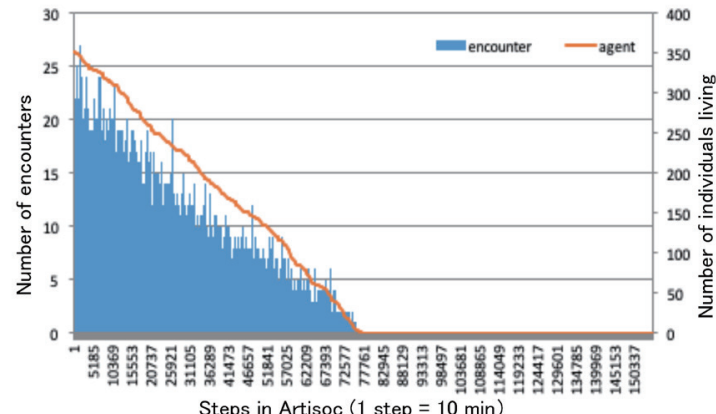

(a)

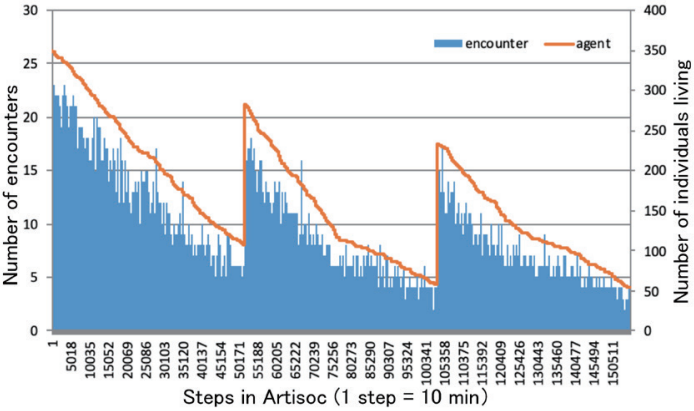

(b)

Fig. 7. (Color online) Numbers of agents and encounters in (a) Method 1 and (b) Method 2.

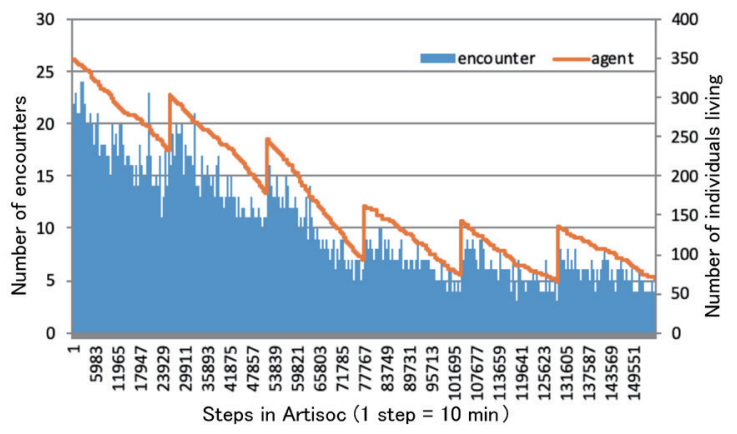

(a)

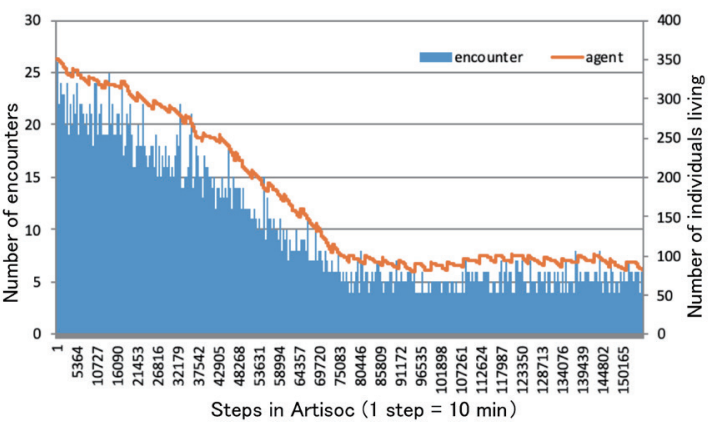

(b)

Fig. 8. (Color online) Numbers of agents and encounters in (a) Method 3 and (b) Method 4. 


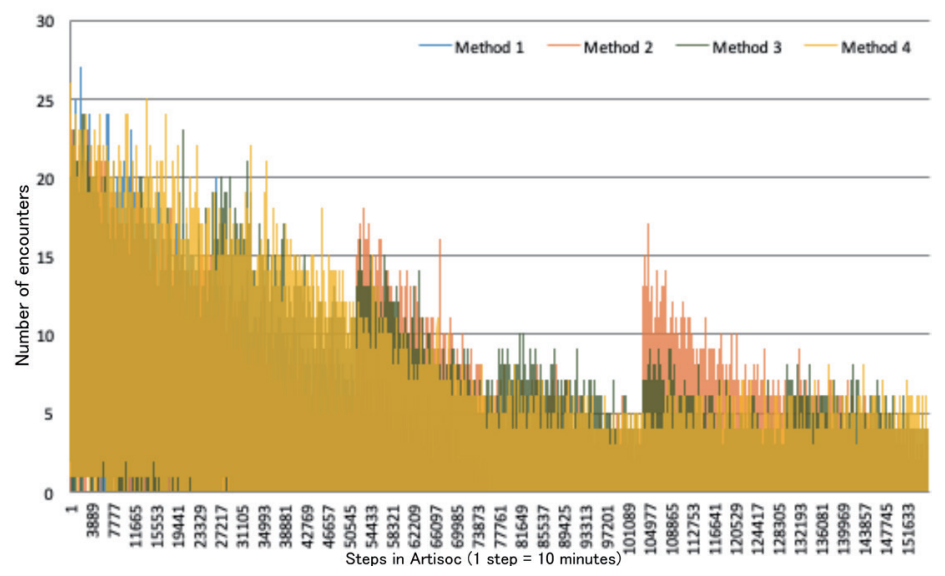

Fig. 9. (Color online) Comparison of encounters for Method 1, Method 2, Method 3, and Method 4.

in the background, the investigation of radiation effects in forest areas around the Fukushima nuclear power plant requires several years to several decades of research to determine the effects of radiation on animals and plants. Therefore, it is necessary to collect environmental information for a long time compared with ordinary field surveys. By conducting a system study considering the lifespan of wildlife individuals, which is our proposed method, the spatial spread of environmental monitoring by animal-to-animal data sharing described in the related research can be realized. The temporal spread of environmental monitoring can also be realized by inheriting the data beyond the generation of wild animals using the proposed method. Therefore, the proposed system is particularly effective when long-term monitoring is required, such as the investigation of radiation effects at remote places where people cannot enter.

\subsection{Findings of field verification in power saving research}

Previous research studies have focused on the power saving of animal-to-animal data sharing and promoted the field trial of the proposal for a wake control method of the communication equipment for power saving. In animal-to-animal data sharing, given that each individual can hold the data of other individuals via wireless communication data transfer, it is assumed that the data collection problem is solved. As discussed in related research, the saving power consumption of wearable devices for long-term monitoring is essential because of the high consumption and limited battery capacity that can be installed to a wild animal. In the field close to the actual wild environment, field trials, such as experiments using four dogs bred at Azabu University and livestock cattle around the Fukushima Daiichi nuclear accident in Figs. 10 and 11, have been conducted to realize power saving, and new findings and issues are being highlighted. For example, similar results could not be obtained in the above two experiments, although the wake control of communication equipment and data transfer between individuals using animal behaviors were attempted to be verified in each field trial for power saving using the same method and equipment. It is suggested as discussed in the related research that the algorithm development was to be based on the premise of dog behavior. 


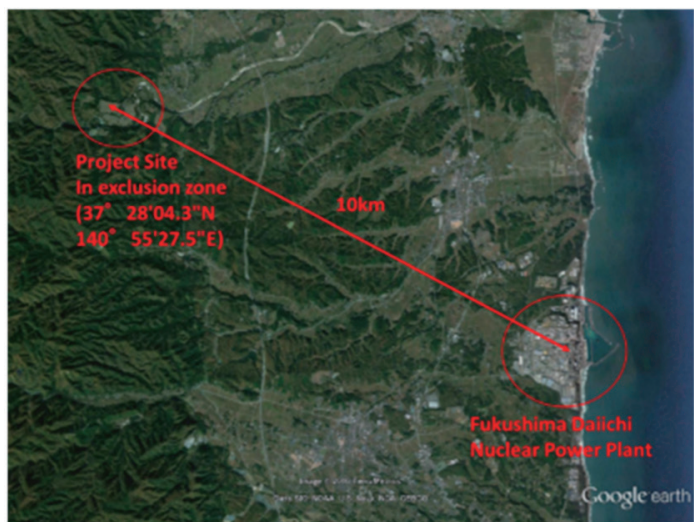

(a)

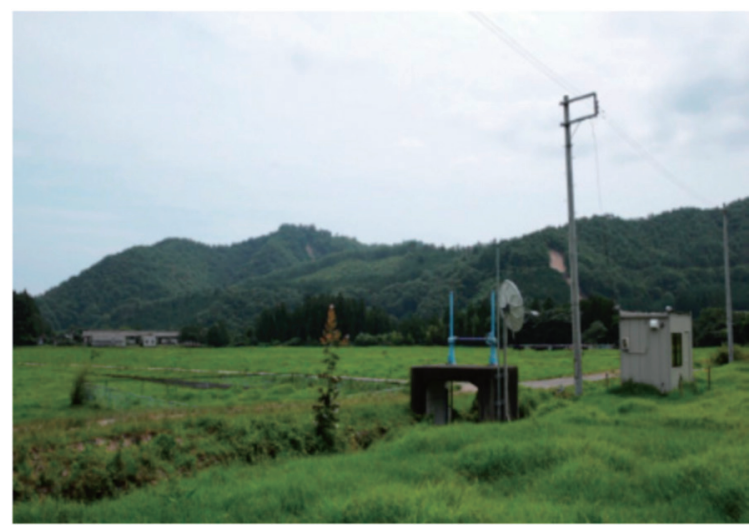

(b)

Fig. 10. (Color online) (a) Project site in the Fukushima exclusion zone (37 28 4.3" N, 14055 27.5" E; 10 km from Fukushima Daiichi Nuclear Power Plant) and (b) sink node station. ${ }^{(7)}$

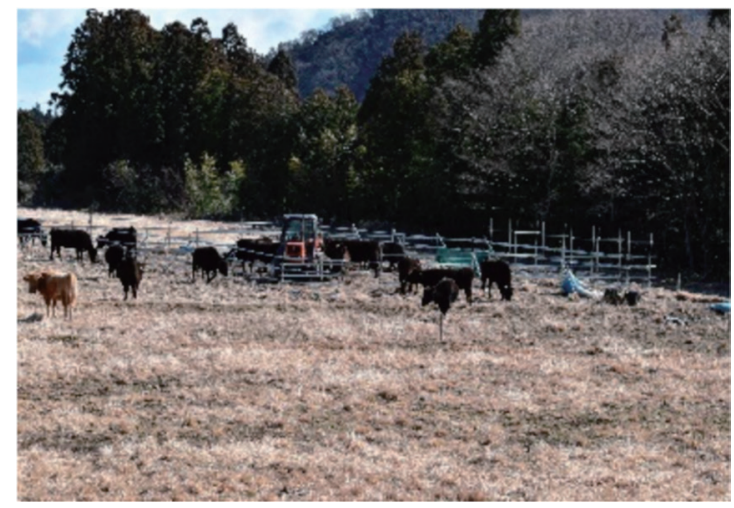

(a)

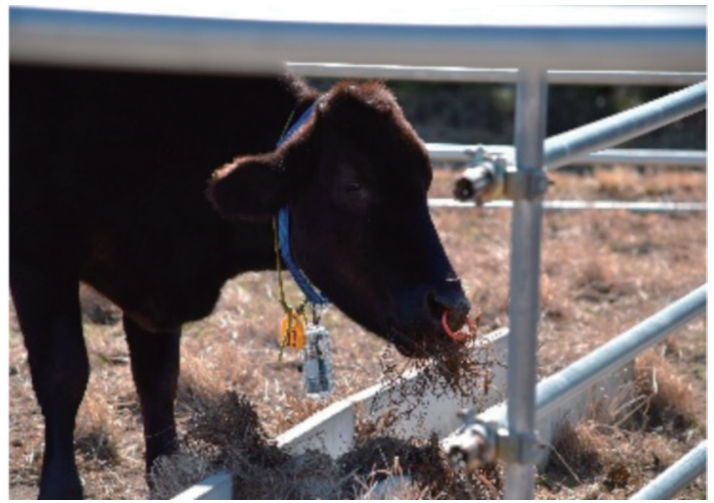

(b)

Fig. 11. (Color online) Animals exposed to surroundings confirmed by camera at the (a) sink node and (b) cage. ${ }^{\text {(7) }}$

Therefore, similar results in each field trial were not obtained because of the difference between a dog and a cow as a target animal. These results show that when wildlife animals are targeted, it is necessary to develop an algorithm that considers the behavior of each target animal by careful observation. Therefore, the research to achieve power saving in the wild environment is continuously required.

\subsection{Workload reduction of ecosystem research using simulation}

Rosatte et al. ${ }^{(11)}$ conducted a field survey in 1994-2007 and captured approximately 150000 raccoons alive. They revealed the density, direct distance, and speed of raccoons in their habitat. Although the information based on these field surveys is useful for clarifying the ecology of raccoons given that long-term surveys for more than a decade require a high personnel cost and a high workload to capture more than 100000 target individuals, the burden on the investigators and the target animals is very high. Moreover, although long-term 
monitoring for more than a dozen years is possible as one project, it is challenging to continue the survey activities across projects because of the high workload. On the other hand, given the significant improvement of information technology, analysis and forecast using simulation based on various types of environmental information are used in various fields. For example, in the field of meteorological observation, observation by manned posts on the ground and by meteorological vessels in the ocean is still being performed. However, by using the large volume of data obtained from unmanned observation, such as stations installed at each site, a balloon called radiosonde, and meteorological satellites, the accuracy of large-scale simulation to forecast from a few hours to several years has significantly improved owing to the significant improvement in the calculation power of the computer. As one of these aspects, adopting a simulation process as one of the observation processes allows the optimization of the resource input and reduces the frequency of manned observations on the ground and observations of meteorological vessels in the sea. As a result, the investigator's workload was reduced. Therefore, by adding the simulation process itself as one of the research methods, resources can be optimized and the necessary information that only exists in the wild environment can be efficiently collected from field research with a reduced workload, such as capturing 150000 raccoons. This could reduce the workload of ecosystem research.

\subsection{Property utilization: Film badge usage and issues}

In this paper, we have proposed a method of obtaining the environmental information of a large area where people cannot get close by attaching a device to wildlife, but there are some other requirements than power saving for wearable devices. Given that this method is supposed to be applied to a large wild environment, it is necessary for the device to be easily attached to many individuals and to have resistance to removal and shock. A film badge ${ }^{(15,16)}$ in Fig. 12 has met the requirements of a wearable material. A film badge is a tool that measures the amount of radiation by utilizing the properties of an object. More specifically, it is a radiation

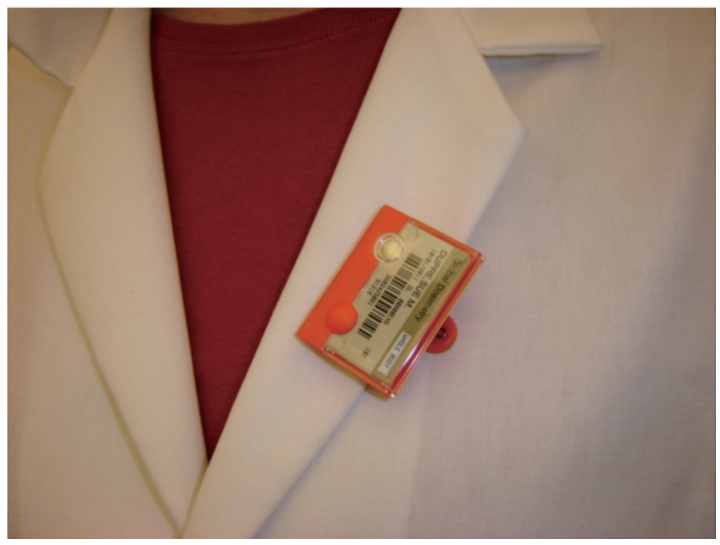

(a)

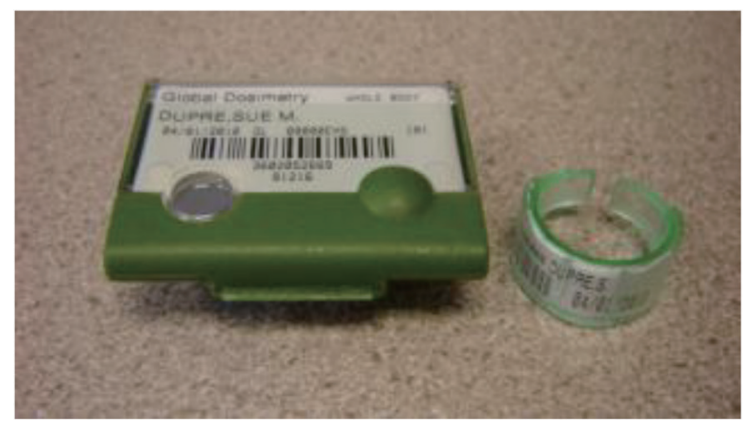

(b)

Fig. 12. (Color online) (a) Nametag on the badge is oriented in the opposite direction from the body and (b) radiation film badge. ${ }^{(16)}$ 
measurement tool utilizing the photosensitizing change of a film by radiation. Owing to the nature of the film badge material, it is possible to measure the amount of radiation and eliminate the need for battery and shock resistance. It can also be easily mounted on many individuals because of its small size, light weight, and low cost. Therefore, the radiation measurement of the forest environment is possible by fulfilling the requirements mentioned above and by attaching a film badge to the wild animal instead of a wearable device. However, there are also problems in research methods using film badges. It is necessary to capture a mounted individual to collect the film badge. Particularly in areas where people cannot enter, such as around the Fukushima nuclear power plant, the return of the mounted individual from the nonaccessible area to the accessible area could not be guaranteed, which makes it challenging to acquire data by individual capture.

To solve these problems, a method combining the animal-to-animal data sharing and the film badge described in this paper can be worth considering. Instead of the wearable device, a film badge is used to convert the dose obtained from the photosensitive action of the film badge into bits, and multi-hop communication is used to transfer data in animal-to-animal data sharing. As an advantage, even with film badges, acquiring data is possible without the need to capture the mounted individual. Moreover, the power consumption of the Geiger counter can be reduced, and the device can be made smaller and lighter. The material change of the film badge is a sensor where the physical principle is applied, and the behavior of a wild animal can be regarded as a moving material. Therefore, by using information technology to connect the animal-to-animal data sharing and the film badge, it is possible to create a value that has never been previously envisioned and enable different approaches to the problem.

\subsection{Engineering use of natural ecosystems}

Okabe ${ }^{(17)}$ stated that it is important to think that society is destined to be created together with people who have lived in the region from the past, according to urban regeneration based on various cases in the EU. She discussed how public space connects the city and its residents. In cities, the relationship between people and their land is deeply linked, and the problem facing the land is a result of past residents' activities. On the other hand, the current residents of the area can only solve the present problem and create a new future. From this idea, if the situation is replaced by a wild environment, the local population will be wild animals that inhabit this area. Owing to their exposure to various threats, such as climate change and alien species, some species must be extinct or their habitat changed, whereas others have adapted to the threat and evolved to continue to settle in the area. Therefore, mounting a wearable device on a wild animal is important for the long-term monitoring of the wild environment given that they have lived in the area for many years and are most aware of the problems in the area where long-term investigation is required because of the impact of radiation. Moreover, the research considering natural habitats, such as animal behavior and individual death since ancient times, is an efficient means for the long-term monitoring of the wild environment, which has different characteristics from the urban environment. This is an example of unprecedented engineering utilizing natural ecosystems. 


\section{Conclusions}

In this paper, we proposed the reproduction of the wild environment using multi-agent simulation and examined the number of individuals introduced at one time and the number of injections to realize data sharing in a group of animals over several years. As a result, we clarified the most efficient method with a high number of encounters that exhibit group behavior despite having a similar workload to the other methods. Therefore, the feasibility and practicability of the proposed method were improved by solving the consideration of the individual's lifespan, which is a problem in animal-to-animal data sharing. Environmental information was successfully collected in an area where power and communication infrastructures do not exist using a low-workload method.

\section{Acknowledgments}

This study was supported by JSPS KAKENHI Grants (18H04089 and 19K22839), the Telecommunications Advancement Foundation, and the Tateisi Science and Technology Foundation.

\section{References}

1 Chernobyl Forum. Expert Group 'Environment'. International Atomic Energy Agency: Environmental Consequences of the Chernobyl Accident and Their Remediation: Twenty Years of Experience; Report of the Chernobyl Forum Expert Group 'Environment' (International Atomic Energy Agency: Vienna, Austria, 2006) p. 137.

2 K. Park, S. Kim, H. Park, and K. Lee: Eng. Struct. 27 (2005) 371. https://doi.org/10.1016/j.engstruct.2004.10.013

3 A. Miyamoto and A. Yabe: JPCS 305 (2011) 1. https://doi.org/10.1088/1742-6596/305/1/012103

4 C. Fiderer, T. Göttert, and U. Zeller: Eur. J. Wildl. Res. 65 (2019) 14. https://doi.org/10.1007/s10344-018-1249-z

5 K. Nakagawa, H. Hill. Kobayashi, and K. Sezaki: Poster. 2014 Augmented Human International Conf. (ACM, 2014) 27. https://doi.org/10.1145/2582051.2582078

6 Y. Kamma, K. Sezaki, and H. Kobayashi: Poster. 2016 Annual International Conf. Mobile Systems, Applications, and Services Companion (ACM, 2016) 40. https://doi.org/10.1145/2938559.2948794

7 H. Kobayashi, K. Nakagawa, K. Makiyama, Y. Sasaki, H. Kudo, B. Niraula, and K. Sezaki: Multimodal Technol. Interact. 2 (2018) 40. https://doi.org/10.3390/mti2030040

8 K. Nakagawa, A. Makita, M. Nagasawa, T. Kikusui, K. Sezaki, and H. Kobayashi: Proc. 2018 Distributed, Ambient and Pervasive Interactions: Understanding Humans (Springer, 2018) 253. https://doi.org/10.1007/9783-319-91125-0 22

9 MAS Community: http://mas.kke.co.jp/ (accessed July 2019).

10 Artisoc 4.2: http://mas.kke.co.jp/modules/tinyd0/index.php?id=17 (accessed July 2019).

11 R. Rosatte, M. Ryckman, K. Ing, S. Proceviat, M. Allan, L. Bruce, D. Donovan, and J. Chris Davies: J. Mammalogy 91 (2010) 122. https://doi.org/10.1644/08-MAMM-A-201R2.1

12 M. Miura: Proc. 2015 3rd Int. Conf. Applied Computing and Information Technology/2nd Int. Conf. Computational Science and Intelligence (IEEE 2015) 242. https://doi.org/10.1109/ACIT-CSI.2015.51

13 Y. Yasuda, N. Mukai, and N. Ishii: Proc. 2013 Second IIAI Int. Conf. Advanced Applied Informatics (IEEE, 2013) 409. https://doi.org/10.1109/IIAI-AAI.2013.66

14 Colorado State University: https://libarts.source.colostate.edu/how-to-keep-visitors-a-safe-distance-fromwildlife-in-national-parks/ (accessed July 2019).

15 National Research Council: Film Badge Dosimetry in Atmospheric Nuclear Tests (The National Academies Press, Washington DC, 1989). https://doi.org/10.17226/1404

16 Princeton University: https://ehs.princeton.edu/laboratory-research/radiation-safety/radiation-monitoringbadges (accessed July 2019).

17 A. Okabe: DBJ Research Center on Global Warming Discussion Paper Series No. 38 (7/2011), https://www.dbj. jp/ricf/pdf/research/DBJ_RCGW_DP38.pdf(accessed August 2019). 